\title{
ANALYSIS OF DYNAMIC MECHANICAL BEHAVIOR IN REVERSE TAYLOR ANVIL-ON-ROD IMPACT TESTS
}

\author{
D. EAKINS, N.N. THADHANI \\ School of Materials Science and Engineering \\ 771 Ferst Drive, Love Mfg. Bld. \\ Georgia Institute of Technology \\ Atlanta, GA 30332, USA
}

\begin{abstract}
A one-dimensional analytical model has been developed for the reverse Taylor anvil-on-rod impact experiment to provide a method of extracting quantitative information from transient deformation states obtained from high-speed camera data. An indication of the plastic wave velocity and dynamic flow stress at varying locations and time are presented for two $\mathrm{Cu}$ OFE specimens impacted at $83 \pm 1$ and $205 \pm 3 \mathrm{~m} / \mathrm{s}$. The results provide supporting evidence of previously reported "non-linear" and "quasi-steady" plastic wave propagation response, as well as a region of perfectly plastic behavior, which is independent of strain-rate in the higher impact velocity experiment. Additionally, the results show good agreement with the average value of dynamic yield stress calculated using Wilkins' analysis, based on a perfectly plastic material response. Furthermore, this work demonstrates the importance of careful design of Taylor impact experiments for
\end{abstract}

Corresponding author. Tel.: +1-404-894-2651.

E-mail address: naresh.thadhani@mse.gatech.edu (N.N. Thadhani). 
minimizing measurement error and capitalizing on perfectly plastic response, for investigating the dynamic mechanical behavior of materials.

Keywords: Reverse Taylor test, plastic wave, dynamic flow strength

\section{Introduction}

A material's mechanical response to dynamic loading is often times explored through the rod-on-rigid-anvil impact test first conducted by G.I. Taylor in 1948 [1]. The test is performed by impacting a cylinder-shaped specimen against a rigid plate, inducing localized plastic deformation at the crushed end. Due to the dynamic nature of the impact event, the strain-rate of deformation encompasses a wide range, which varies along the specimen length and radius, as well as over time. Consequently, while a single Taylor impact experiment is rich with constitutive information, the challenge has been to interpret the resulting data in ways that are meaningful.

Attempts to quantify material response from recovered Taylor impact specimens have led to the development of several one-dimensional models [1-4], which seek to describe the flow stress of the material as a function of overall changes in dimensions. This approach resulted primarily from the inability to monitor the specimen profile as it evolved during deformation. As a result, these early models subscribe to the concept of a largely singular value of the dynamic yield strength for a given strain-rate regime.

This limitation has been overcome with the use of high-speed photography, first incorporated by SRI international in conducting Taylor impact experiments [5]. Capture of the specimen profiles during deformation provides time-resolved information against which theoretical models can be validated. Additionally, the transient images can be used to 
determine stress and strain states at several stages during the impact event. The latter was demonstrated by House et al. [6] with a procedure for deducing stress along the specimen length as a function of time. In this work, camera data obtained from rod-on-rigid-anvil Taylor tests were used for measurements of instantaneous radial strain along the rod length and iso-strain plastic wave locations. By extending Taylor's original analysis, the stresses could then be determined from the iso-strain history. Results indicated that the instantaneous flow stress depends upon strain rate, and is within the same regime as the value predicted from Taylor's analysis using end state measurements made on recovered samples. It was acknowledged by the authors however, that the time between frames chosen for the depicted study was large, and consequently insensitive to any small-scale nonlinear deformation behavior.

High-speed photography has also led to more complete descriptions of plastic wave propagation at high strain-rates. Following Taylor tests conducted on $\mathrm{OFHC} \mathrm{Cu}$, three regimes of distinct plastic wave behavior, referred to as Phases I-III, have been suggested by Jones et al. [7, 8]. At early times (Phase I), the propagation of the plastic/elastic boundary is "non-linear" (varying velocity). This is followed by a "quasi-steady" regime of constant plastic wave front velocity in Phase II, succeeded by Phase III, in which the plastic wave amplitude falls below the yield limit and deformation is arrested. Thus far, these observations have only been made for the leading deformation wave, and the behavior of the following iso-strain waves has not been considered.

In recent years, the desire to utilize multiple time-resolved measurement techniques has prompted the use of an alternate impact test configuration, one which allows the specimen to remain fixed. This requirement is accomplished in the reverse Taylor anvil-on-rod 
impact experiment, whereby a stationary rod-shaped specimen is impacted by a rigid-faced projectile [9]. In contrast to the original Taylor (rod-on-anvil) test, the deformation event in the reverse Taylor (anvil-on-rod) test configuration does not remain fixed to any laboratory reference frame. Consequently, images of the deformation event captured using stationary high-speed photography do not contain the same perspective or fixed boundaries, making application of the House analysis troublesome. Thus, an alternative analysis specific to the reverse configuration is needed.

In the current investigation, reverse Taylor anvil-on-rod impact experiments are conducted on oxygen-free electronic grade $\mathrm{Cu}$ specimens at 83 and $205 \mathrm{~m} / \mathrm{s}$ utilizing highspeed digital photography to capture images of the intermediate deformation states. A

revised form of the House analysis [6] developed for the reverse configuration is applied to the specimen profiles to expand the description of plastic wave propagation presented by Jones et al. [7], and to estimate the plastic wave velocity and dynamic flow stress as a function of axial position and time.

\section{Experimental Procedure}

\subsection{Reverse Taylor anvil-on-rod impact test set-up}

The experiment considered in the current analysis is commonly referred to as the reverse Taylor anvil-on-rod impact test, shown schematically in Figure 1. A cylindrical specimen is held by a thin PMMA support disc, mounted to a 2-axis adjustment ring fixed to the end of an $80 \mathrm{~mm}$ diameter helium-driven gas gun. A rigid steel flyer backed by a 7" aluminum sabot is fired in the range of $50-250 \mathrm{~m} / \mathrm{s}$ using a wrap-around breech gas delivery system. Impact between the specimen and anvil (flyer) is recorded by an Imacon 
200 high-speed digital framing camera, triggered by end impact crush pins preceding the specimen by approximately $2 \mathrm{~mm}$. A soft-catch tank is present following impact to avoid secondary damage to the specimen and allow full recovery. A complete summary of the specimen preparation and experiment setup is presented elsewhere [10].

Two experiments were conducted on OFE $\mathrm{Cu}$ specimens observing initial dimensions of $\sim 19 \mathrm{~mm}$ diameter and $\sim 75 \mathrm{~mm}$ length at nominal impact velocities of 83 and $205 \mathrm{~m} / \mathrm{s}$. Images of the recovered $\mathrm{Cu}$ specimens tested at these velocities are shown in Figure 2. The specimen tested at $205 \mathrm{~m} / \mathrm{s}$ shows evidence of barreling shortly behind the front impact zone, characteristic of high-velocity impact experiments, while the $83 \mathrm{~m} / \mathrm{s}$ sample shows more uniform front end deformation. The two tests at these velocities provide typical examples of variations in deformation generally observed in Taylor tests.

\subsection{Analysis of elastic/plastic transition front}

The method of deducing stress and strain data from camera images of Taylor impact experiments developed by House et al. [6] was modified for the reverse Taylor configuration employed in the present work. It should be pointed out that while the mechanics of deformation are identical between the two configurations (standard versus reverse), the model is more easily derived using an alternate choice of reference frame that matches the experimental conditions. Consider the schematic in Figure 3, showing two stages (at times $t_{1}$ and $t_{2}$ ) in the deformation of a Taylor specimen tested in the reverse anvil-on-rod configuration. At $t_{1}$ a rigid anvil has impacted the stationary rod-shaped specimen at a velocity $u$ producing an elastic wave propagating at velocity $C$, and elastic/plastic transition front traveling at a velocity $v$. The respective elastic wave and rigid 
anvil velocities, $C$ and $u$, are assumed to be constant, while the elastic/plastic transition front velocity is expected to vary.

The rod is divided into three portions separated by the fronts and differing in density or cross-sectional area. $U_{p 1}$ and $U_{p 2}$ are considered as residual particle velocities behind the elastic and elastic/plastic transition fronts, respectively, while the particle velocity in the undisturbed region (ahead of the elastic front) is assumed zero. Also shown is the specimen at a later time, given by $t_{2}$, illustrating the advanced positions of the anvil, and the corresponding elastic, and elastic/plastic transition fronts. In each case, the front velocities are measured with respect to the back free surface, which remains stationary until the arrival of the elastic wave front. The elastic/plastic transition velocity, $v$, is defined based on its position at times $t_{1}$ and $t_{2}$, relative to the stationary back surface. The position of the elastic/plastic transition front with respect to the back surface at $t_{1}$ is $x_{1}$. At $t_{2}$ the elastic/plastic front has moved to $x_{2}$. Hence, the elastic/plastic transition front velocity is defined as:

$$
v=\frac{x_{1}-x_{2}}{t_{2}-t_{1}}
$$

Similar to Taylor's analysis, an expression for the stress and strain may be derived using the conservation equations applied across the respective elastic and elastic/plastic transition fronts. It should be noted that in all cases when conservation laws of mass and momentum are used, the equalities are performed between adjacent time-frames, rather than at a particular instance in time. 
At the elastic boundary, the rate of mass flow entering the elastic front must equal the rate of mass flow leaving the front; Hence,

$$
\begin{gathered}
\frac{d}{d t}\left(M^{\text {in }}\right)=\frac{d}{d t}\left(M^{\text {out }}\right) \\
\frac{d}{d t}\left(\rho_{0} A_{0} C d t\right)=\frac{d}{d t}\left[\rho A_{0}\left(C-U_{p 1}\right) d t\right] \\
\rho_{0} A_{0} C=\rho A_{0}\left(C-U_{p 1}\right)
\end{gathered}
$$

where, $M^{\text {in }}, M^{\text {out }}, A_{0}, \rho_{0}$, and $\rho$ are respectively the mass entering the front, mass leaving the front, original cross-sectional area, starting density, and elastically strained density. This expression contains the unknown residual particle velocity behind the elastic wave front $U_{p 1}$, which may be expressed in terms of the longitudinal elastic wave front velocity $C$ as:

$$
U_{p 1}=C\left(\frac{\rho-\rho_{0}}{\rho}\right)
$$

Applying the conservation of mass across the elastic/plastic transition front gives an equivalent expression for the rate of mass flow entering and exiting the front using Equation 2a:

$$
\begin{aligned}
\frac{d}{d t}\left[\rho A_{0}\left(v-U_{p 1}\right) d t\right] & =\frac{d}{d t}\left[\rho A\left(v-U_{p 2}\right) d t\right] \\
\rho A_{0}\left(v-U_{p 1}\right) & =\rho A\left(v-U_{p 2}\right)
\end{aligned}
$$


where, $v$ represents the speed at which the elastic/plastic transition front (leading plastic wave) moves into elastically strained material, and $A$ is the increased cross-sectional area just within the plastic zone. Again, the initial area $A_{0}$ is taken at a position ahead of the elastic/plastic transition front at $t_{1}$, while the strained area $A$ is taken at that same position after the front has passed at a later time $t_{2}$.

Rearranging Equation $4 \mathrm{~b}$ gives an expression for $U_{p 2}$ in terms of $U_{p 1}$ :

$$
U_{p 2}=v-\frac{A_{0}}{A}\left(v-U_{p 1}\right)
$$

Inserting the expression for $U_{p 1}$ from Equation 3 into Equation 5 gives,

$$
\begin{aligned}
& U_{p 2}=v-\frac{A_{0}}{A}\left[v-C\left(\frac{\rho-\rho_{0}}{\rho}\right)\right] \\
& \quad \text { or, } U_{p 2}=v\left(\frac{A-A_{0}}{A}\right)+C \frac{\left(\rho-\rho_{0}\right) A_{0}}{\rho A}
\end{aligned}
$$

Now consider the conservation of momentum across the elastic/plastic transition front, by equating the rate of change in momentum to the net impulse. The impulse may be expressed as,

$$
F d t=\sigma\left(A_{0}-A\right) d t
$$


which in rate form is,

$$
F=\sigma\left(A_{0}-A\right)
$$

where, $F$ is force, $\sigma$ is stress, and the terms $A$ and $A_{0}$ are respectively the initial and strained cross-sectional areas, as defined earlier. Next, the rate of momentum change across the elastic/plastic transition front can be written as,

$$
\frac{d}{d t}\left(p^{\text {in }}-p^{\text {out }}\right)=\frac{d}{d t}\left(M^{\text {in }} v^{\text {in }}-M^{\text {out }} v^{\text {out }}\right)
$$

where, $p$ is the momentum, $M$ and $v$ are the mass and associated velocity of material entering or exiting the transition front. Using the expressions for mass derived previously (Equation 4a) for the conservation of mass across the elastic/plastic front, Equation 8a becomes:

$$
\frac{d}{d t}\left(p^{\text {in }}-p^{\text {out }}\right)=\frac{d}{d t}\left[\rho A_{0}\left(v-U_{p 1}\right) v^{\text {in }} d t-\rho A\left(v-U_{p 2}\right) v^{\text {out }} d t\right]
$$

which, since $v^{\text {in }}=\left(v-U_{p 1}\right)$ and $v^{\text {out }}=\left(v-U_{p 2}\right)$, simplifies to,

$$
\frac{d}{d t}\left(p^{i n}-p^{o u t}\right)=\rho A_{0}\left(v-U_{p 1}\right)^{2}-\rho A\left(v-U_{p 2}\right)^{2}
$$


Thus, equating Equations $7 \mathrm{~b}$ and $8 \mathrm{c}$ to apply the conservation of momentum across the elastic/plastic transition boundary yields:

$$
\sigma\left(A_{0}-A\right)=\rho A_{0}\left(v-U_{p 1}\right)^{2}-\rho A\left(v-U_{p 2}\right)^{2}
$$

Replacing $U_{p 2}$ and $U_{p 1}$ with the quantities expressed in Equations 3 and $6 \mathrm{~b}$ in the above relationship,

$$
\sigma\left(A_{0}-A\right)=\rho A_{0}\left[v-C\left(\frac{\rho-\rho_{0}}{\rho}\right)\right]^{2}-\rho A\left[v-v\left(\frac{A-A_{0}}{A}\right)-C\left(\frac{\left(\rho-\rho_{0}\right) A_{0}}{\rho A}\right)\right]^{2}
$$

one obtains,

$$
\sigma\left(A_{0}-A\right)=\frac{A_{0}}{\rho}\left(\frac{A-A_{0}}{A}\right)\left[v \rho-C\left(\rho-\rho_{0}\right)\right]^{2}
$$

Considering the density change across the elastic front to be negligible (i.e., $\rho \cong \rho_{0}$ ),

Equation 11 simplifies to the following form:

$$
\begin{aligned}
& \sigma\left(A_{0}-A\right)=\frac{A_{0}}{\rho_{0}}\left(\frac{A-A_{0}}{A}\right)\left(v \rho_{0}\right)^{2} \\
& \text { or, } \sigma=-\frac{\rho_{0} A_{0} v^{2}}{A}=-\rho_{0} v^{2}(1-\varepsilon)
\end{aligned}
$$




$$
\text { where, } \varepsilon=\frac{A-A_{0}}{A}
$$

Equations 13 and 14 define the stress and strain relationships averaged over two frames for the elastic/plastic transition front propagating at velocity $v$, defined in Equation 1. Note that unlike the similar derivations by Wilkins [3] or House [11], the formerly assumed constant back-end speed of the rod does not appear in Equation 13. If the change in backend speed is to be considered (due to momentum transfer and acceleration in the reverse impact configuration), a shift in reference point must be accounted for, or alternatively, the analysis would only be valid until such time when the back free surface sets into motion with the arrival of the elastic wave front.

\subsection{Analysis of iso-strain plastic wave fronts}

The usefulness of high-speed digital photography is in the ability to capture the instantaneous position of not only the elastic/plastic transition front, the focus of the derivation described above, but of also the varying degrees of strain throughout the plastic zone. As a result, this technique can provide instantaneous measures of areal strain and velocity, which in turn can be used to deduce the stress at any axial location within the plastic zone. An example of how these measures are obtained is illustrated schematically in Figure 4, which plots the radial profile (half-section) of the plastically deformed region (shown in Figure 3 ) at time $t_{1}$ and $t_{2}$. At an arbitrary location within the plastic zone, the incremental areal strain is evidenced by the increase in radius from $R_{1}$ (at time $t_{1}$ ) to $R_{2}$ (at time $t_{2}$ ). The velocity of this strain level (iso-strain) is determined from its position at the earlier time, $t_{1}$ i.e., 


$$
v^{\prime}=\frac{x_{1}^{\prime}-x_{2}^{\prime}}{t_{2}-t_{1}}
$$

where, $x_{1}$ ' and $x_{2}$ ' are the positions of the iso-strain front at their respective times, referenced from the back surface. To determine the stress and strain relationship, one can apply the conservation of mass and momentum across any such iso-strain front in the plastic deformation zone, instead of the elastic/plastic transition front, and show that,

$$
\begin{array}{r}
\sigma=-\rho_{0}\left(v^{\prime}-U_{p 2}\right)^{2}\left(1-\varepsilon^{\prime}\right) \\
\text { where, } \varepsilon^{\prime}=\frac{A_{2}-A_{1}}{A_{2}}=\frac{R_{2}^{2}-R_{1}^{2}}{R_{2}^{2}}
\end{array}
$$

The $U_{p 2}$ term in Equation 16 is the particle velocity behind the elastic/plastic transition front (defined earlier, Figure 3). It should be noted that unlike the work of House et al. [6], the areal strain in Equation 17 is an incremental measure which, coupled with the incremental iso-strain front velocity, gives an expression for the true stress (Equation 16). The concerns regarding the motion of the back surface at later times are relieved, as it is considered that the reflected elastic wave has negligible effect on the iso-strain wave propagation in the plastic zone. On the other hand, the appearance of $U_{p 2}$ complicates this expression, requiring assumptions to be made about the particle velocity in the plastic region (usually ignored). With this limitation in mind, the current analysis is useful for comparing the dynamic plastic deformation response of different materials or the material response at different impact velocities, in cases where transient deformation states, captured by imaging with a high-speed camera, are available. Hence, the rationale for the work 
presented in this paper is to utilize transient deformation states, obtained via high-speed digital imaging, to describe the plastic deformation response based on the variation of plastic wave velocity and the stress required to produce incremental deformation as a function of axial position and time.

\section{Results and Discussion}

Selected high-speed digital camera images recorded from the two reverse Taylor impact experiments performed at 83 and $205 \mathrm{~m} / \mathrm{s}$ are shown in Figure 5 and Figure 6, respectively. The resolution of the images is based upon the field of view, and was 12.3 and 9.7 pixels/mm for the low (Figure 5) and high velocity (Figure 6) shots, respectively. The images were captured from slightly different perspectives, which depended on the choice of frame center. At the lower velocity the field of view was centered on the specimen back surface, rather than on the specimen mid-length as in the higher velocity experiment. This minimized the projected width of the specimen support disc, and at the same time eliminated curvature from the back surface. Since the impact plane at $83 \mathrm{~m} / \mathrm{s}$ exhibited mirroring of the specimen, the peak diameter was used to locate the crush plane.

The analytical method described in the previous section allows the stress to be estimated from measurements of the iso-strain plastic wave velocities. The image analysis and profile measurement techniques employed were similar to those described in the paper by House et al. [6]. The analysis requires precise measurement of specimen radius as a function of axial length. Due to blurring of the specimen boundaries, an uncertainty of as much as 5 pixels $(405 \mu \mathrm{m}$ at $83 \mathrm{~m} / \mathrm{s}$, or $515 \mu \mathrm{m}$ at $205 \mathrm{~m} / \mathrm{s})$ was observed. It is also important to note that the referenced positions are not true Lagrangian descriptions. As 
such, referential inaccuracies arise that are minimized by selecting frames temporally close to one another. On the other hand, choosing frames separated by too short a time only increases the probability of profile overlapping. Thus, special care was taken in the timing and selection of each frame. For each experiment, five frames were chosen spaced regularly apart in time to prevent overlapping of the specimen profiles caused by measurement uncertainties and to reduce the Lagrangian uncertainty. Two later frames were also included for the higher velocity experiment. The interframe times between the images selected for analysis for both experiments are listed in Table 1.

Measurements of the specimen diameter were performed for every pixel along the specimen's length. By assuming symmetric deformation, the radius as a function of position was taken to be half the measured diameters. In the case of non-symmetric deformations, this assumption is not valid, and has the effect of averaging over the differing radii. The areal strains were calculated next using Equation 17, and are plotted as a function of axial length for the $83 \mathrm{~m} / \mathrm{s}$ and $205 \mathrm{~m} / \mathrm{s}$ experiments, as shown in Figure 7(a) and (b), respectively. Also shown are the polynomial functions used to fit the data which allow the tracking of an arbitrary strain's position as a function of time. Using the approach described in the previous section, the iso-strain plastic wave velocities (Equation 15) were calculated for 0.05 to 0.36 strain in the case of the $83 \mathrm{~m} / \mathrm{s}$ experiment, and for 0.05 to 0.55 strain in the case of the $205 \mathrm{~m} / \mathrm{s}$ experiment. It is important to note that the velocities calculated are averages over the interframe time and hence, are not expected to capture any non-linear behavior within this time interval.

Finally, the areal strain-rate as a function of axial position for a strain level of 0.11 to 0.35 in the case of the high velocity experiment is shown in Figure 8. As expected, the 
specimen is subjected to a range of strain-rates, which decrease with the propagation of the plastic wave along the rod length. For the $205 \mathrm{~m} / \mathrm{s}$ specimen, strain-rates up to $\sim 3 \times 10^{4} \mathrm{~s}^{-1}$ are observed at the impact face, dropping into the $10^{3} \mathrm{~s}^{-1}$ regime about $55 \mathrm{~mm}$ from the back end of the $75 \mathrm{~mm}$ long sample. Once more, it must be reminded that the current analysis lacks the ability to maintain a pure Lagrangian reference, which has the largest influence on the strain-rate calculations in the reverse configuration. Nevertheless, the qualitative significance of these observations remains unchanged. Similar strain-rate versus distance constructions for the low velocity experiment are omitted, since the uncertainty in profile measurements at low strains greatly influences the characteristics of these plots, although the trend of the range of strain rates $\left(7 \times 10^{3}\right.$ to $\left.1 \times 10^{3} \mathrm{~s}^{-1}\right)$ decreasing with plastic wave propagation along the rod length remains the same.

\subsection{Iso-strain Plastic Wave Velocity Variation}

A plot showing the change in iso-strain plastic wave velocity with increased distance along the specimen length is presented in Figure 9 for several strain values ( 0.11 to 0.26 for both experiments). Since the curves are derived from the same material tested at two different impact velocities, they illustrate the influence of strain-rate on plastic wave propagation. Overall, the specimen tested at $83 \mathrm{~m} / \mathrm{s}$ contains slower plastic wave velocities than the $205 \mathrm{~m} / \mathrm{s}$ sample. This is not unexpected, since the plastic wave speed scales with impact velocity [8]. The data from the $205 \mathrm{~m} / \mathrm{s}$ sample also exhibits a plateau in wave speed shortly after impact. This region is consistent with the Phase II deformation described by Jones et al.[8], characterized by quasi-steady (constant velocity) wave propagation. The onset of the iso-strain velocity plateau $\sim 25 \mu$ s after impact is also within the same order of 
magnitude as the $10 \mu$ s transition time reported by Jones et al. [8] for OFHC Cu tested at a similar velocity; the small displacement in time may be due to slight differences in material and initial diameter. Elsewhere, the iso-strain velocity contours for several strain values appear to slow at a similar rate, independent of their magnitude.

It should be noted that in addition to the velocity of the leading deformation wave, the current analysis also offers a description of wave propagation for the iso-strain fronts that follow the leading plastic wave. While similar characteristics are observed in the subsequent waves, the boundary between Phase I and II propagation becomes increasingly diminished. This plateau is seemingly absent from the lower velocity experiment.

It is important to note that the resolution of these curves is dependent upon the number of frames chosen for the analysis. While each iso-strain curve can be further refined by using more tightly spaced frames, they would also suffer from increased measurement error. Furthermore, the experiments shown appear to capture different stages in the deformation event; the $205 \mathrm{~m} / \mathrm{s}$ test concentrates on behavior early during deformation (Phases I and II), while the $83 \mathrm{~m} / \mathrm{s}$ test reveals the final stages of wave propagation (Phase III). Thus, while a plateau is not observed in the lower velocity test, it is possible that the frame spacing and window were not optimal to observe this behavior. Alternatively, the three phase description of plastic wave propagation may not apply below a certain critical impact velocity.

The origin of the Phase II plateau might possibly be explained from observation of the transient images and the final recovered sample. Examination of the final deformation profiles of recovered samples shown in Figure 2 reveals that only the high velocity specimen shows evidence of barreling preceding the flared impact zone. While this region 
is not reflected in the transient images, its development is evidenced by the change in slope of the profiles of strain shown in Figure 7. This change, from a concave to convex profile, occurs at 25 to $35 \mu$ s after impact, approximately the same time as the transition from Phase

I to II. Under this same argument, the absence of a plateau in the low velocity experiment can be explained to be due to insufficient impact velocity to induce concave deformation at early times, which results in the ever convex shape of the transient profiles seen in Figure 5.

\subsection{Variation in Flow Stress}

Figure 10 presents the stress, calculated using Equations 16 and 17, required to produce incremental deformation as a function of distance in the plastically deformed region near the impact face for the same set of strain values. Again, since the plots are constructed for two different impact velocities, the curves provide a qualitative indication of the strain-rate dependence of dynamic yield stress. In the data presented, the strain history curves for $\varepsilon \leq$ 0.05 are omitted, since these start only several millimeters into the sample. Similar to the velocity calculations, the stresses calculated are averaged between frames, and consequently are conservative estimates of stress. For the $83 \mathrm{~m} / \mathrm{s}$ sample, it can be seen that the stress necessary to produce increasingly greater amounts of strain decreases similarly at different positions along the axial length of the rod. On the other hand, data from the 205 $\mathrm{m} / \mathrm{s}$ specimen contains a plateau (similar to that observed in the velocity plot in Figure 9) centered at about $800 \mathrm{MPa}$. In this region, all strain in the range shown occurs at nearly the same stress level, albeit at varying strain rates. The precise extent of this region is not immediately obvious from the current plot however, and is complicated by the varying position of strained material with respect to the back end. Nevertheless, the results illustrate 
a plastic deformation region in the reverse Taylor impact sample over which flow stress appears to be constant, and independent of strain rate.

The average dynamic flow stress calculated from overall length change using Equation 3 in the paper by Wilkins [3], for both tests is also included in Figure 10. Though the data shows that it would be difficult to select a single value for the dynamic flow strength, the value of the Wilkins stress falls very close to both experimental data sets. It would be of interest to see if the Wilkins dynamic flow stress is an average of a complete data set (Phase I through Phase III), if these phases can be captured in a single experiment, performed at a certain critical impact velocity.

The current method can be greatly improved through the use of a Lagrangian particle tracking technique, for instance speckle photography or interferometry $[12,13]$. Such a technique is essential to reduce the uncertainty in plastic wave location, a critical variable in the derivation of the dynamic flow stress. However, the results of the present work clearly illustrate that it is important to take these issues into consideration when designing experiments of this type, i.e. choosing an appropriate impact velocity and framing timeline to capture the entire deformation history of a Taylor specimen. Otherwise, the errors caused by measurement uncertainty will override any phenomenon of interest. The cut-off impact velocity below which measurement errors become substantial will vary according to the imaging equipment resolution.

It should be noted that the purpose of the one-dimensional analysis presented in this paper is to obtain a better insight into the stress-strain response of the plastically deformed region of a Taylor-impact test sample, and not necessarily to generate stress-strain curves. Computational simulations employing constitutive equations based on physical models can 
certainly be used to account for interactions with lateral boundaries and obtain dynamic stress-strain curves, by correlating simulated deformation profiles with experimentallyrecorded transient patterns obtained with a high-speed camera. The results of such an analysis, however, would depend upon the validity of the constitutive model used, and particularly on its adherence to the transient (and not just final) deformation states. The results of such validation tests performed on the copper samples presented in this paper are described elsewhere [10]. The approach presented in the current paper provides a onedimensional analysis for estimating the flow stress within a deforming Taylor test specimen directly from experiments in which high speed imaging is used to capture transient deformation states.

\section{Conclusions}

A simplistic one-dimensional analytical model for estimating plastic wave behavior and dynamic flow stress using high-speed photographic images has been developed for the reverse Taylor anvil-on-rod impact configuration. The model has been demonstrated using time-resolved transient deformation profiles obtained from experiments conducted at 83 and $205 \mathrm{~m} / \mathrm{s}$ on OFE Cu specimens. Results suggest that a three phase description of plastic wave propagation consisting of varying (Phase I), constant (Phase II), and arresting (Phase III) plastic wave velocity, only applies to $\mathrm{Cu}$ specimens impacted at sufficiently high velocities. The onset of Phase II propagation appears linked to the shape of the transient profiles. At the low impact velocity, the transient profiles are convex throughout the deformation event, and do not develop a barreled region. In Phase II for the high velocity experiment, the dynamic flow stresses calculated as a function of time are centered at about 
$800 \mathrm{MPa}$, irrespective of strain level, suggesting a rate-independent perfectly plastic material response. Comparison of the deformation response between the two experiments (at $83 \mathrm{~m} / \mathrm{s}$ and $205 \mathrm{~m} / \mathrm{s}$ ) reveals the importance of frame timing, equipment resolution, and impact velocity when probing plastic wave behavior in a Taylor impact specimen.

\section{Acknowledgements}

The authors acknowledge the support provided by AFOSR Grant No. F49620-02-10382. The NDSEG fellowship awarded to Dan Eakins is also acknowledged. 


\section{References}

1. Taylor G. The use of flat-ended projectiles for determining dynamic yield stress. I. Theoretical considerations. In: Proceedings of the Royal Society of London. The Royal Society,1948. p. 289-299.

2. Whiffin AC. The use of flat ended projectiles for determining dynamic yield stress. Ii. Tests on various metallic materials. In: Proceedings of the Royal Society of London. The Royal Society,1948. p. 300-322.

3. Wilkins ML and Guinan MW. Impact of cylinders on a rigid boundary. J Appl Phys. 1973; 44 (3):1200-1206.

4. Hawkyard JB. A theory for the mushrooming of flat-ended projectiles impinging on a flat rigid anvil, using energy considerations. Int J Mech Sci. 1969; 11:313-333.

5. Erlich DC and Shockey DA, Dynamic flow curve of 4340 steel as determined by the symmetric rod impact test, Shock waves in condensed matter, Elsevier Science Publishers, 129-132, (1983).

6. House JW, Aref B, Foster JC and Gillis PP. Film data reduction from taylor impact tests. Journal of Strain Analysis. 1999; 34 (5):337-345.

7. Jones SE, Gillis PP, Foster JC, Jr. and Wilson LL. One-dimensional, two-phase flow model for taylor impact specimens. Journal of Engineering Materials and Technology, Transactions of the ASME 1991; 113 (2):228.

8. Jones SE, Maudlin PJ and Foster JC, Jr. An engineering analysis of plastic wave propagation in the taylor test. Int J Impact Engng 1997; 19 (2):166.

9. Gust WH. High impact deformation of metal cylinders at elevated temperatures. J Appl Phys. 1982; 53 (5):3566-3575.

10. Eakins DE. Reverse taylor anvil-on-rod impact test for validating applicability of standard strength models to transient deformation states. J Appl Phys. to be published Oct, 2006;

11. House JW, Lewis JC, Gillis PP and Wilson LL. Estimation of flow stress under high rate plastic deformation. Int J Impact Engng. 1995; 16 (2):189-200.

12. Asundi A. Sampled-speckle photography for measurement of deformation. Optics Letters. 2000; 25 (4):218-220.

13. Davila A, Huntley JM, Kaufmann GH and Kerr D. High-speed dynamic speckle interferometry: Phase errors due to intensity, velocity, and speckle decorrelation. Applied optics. 2005; 44 (19):3954-3962. 


\section{Figure Captions}

Figure 1. Schematic of the reverse Taylor anvil-on-rod impact experiment set-up showing the configuration of the projectile, sample, diagnostics, and soft-recovery.

Figure 2. Recovered OFE Cu Taylor specimens ( originally $\sim 19 \mathrm{~mm}$ diameter and $\sim 75$ $\mathrm{mm}$ length ) tested at 205 and $83 \mathrm{~m} / \mathrm{s}$. Note the barreled region preceding the flared end in the higher velocity experiment, absent in the specimen tested at the lower velocity.

Figure 3. Intermediate stages (at time $t_{1}$ and $t_{2}$ ) in the deformation of a cylindrical specimen impacted in the reverse configuration identifying the variables used in the current analysis. Impact proceeds from the left, while the front velocities $(u, v, C)$, and particle velocities $\left(U_{\mathrm{p} 1}, U_{\mathrm{p} 2}\right)$, are referenced from the back free surface.

Figure 4. A plot of the plastic zone cross-section of a reverse Taylor specimen at two times $\left(t_{1}\right.$ and $\left.t_{2}\right)$, showing the measures used to determine the incremental strain and isostrain front velocity. The strain is associated with the change in area from $A_{1}$ to $A_{2}$, while the velocity follows from the positions of area $A_{2}$ at times $t_{1}$ and $t_{2}$.

Figure 5. Selected high-speed digital images capturing the deformation of the $\mathrm{Cu}$ rod impacted at $83 \mathrm{~m} / \mathrm{s}$; times shown are referenced from impact. The back end remains motionless until up to $71 \mu \mathrm{s}$ after impact. Crushing of the trigger pins is also seen in the frames. 
Figure 6. Selected transient deformation profiles of the experiment conducted at 205 $\mathrm{m} / \mathrm{s}$; times shown are referenced from impact. The back end remains stationary until up to $45 \mu \mathrm{s}$ after impact.

Figure 7. Radial strain plots at various stages of deformation referenced from the specimen back-end, generated from high-speed camera images for (a) $83 \mathrm{~m} / \mathrm{s}$ (b) 205 $\mathrm{m} / \mathrm{s}$ experiment. The specimen tested at $205 \mathrm{~m} / \mathrm{s}$ undergoes far greater deformation $(\sim 80 \%)$, and exhibits a change in profile slope about $35 \mu$ s after impact.

Figure 8. Incremental strain-rate for various iso-strain fronts in the $205 \mathrm{~m} / \mathrm{s}$ experiment, exhibiting an overall decrease with increased plastic wave propagation depth. The strain-rate at the impact face at early times is within the $10^{4} \mathrm{~s}^{-1}$ regime, and falls to within the $10^{3} \mathrm{~s}^{-1}$ regime as deformation continues.

Figure 9. Plot of iso-strain plastic wave velocities as a function of axial length. Note the regime of nearly constant wave velocity in specimen tested at higher velocity, characteristic of Phase II behavior.

Figure 10. Plot of stress required to produce incremental strains as a function of axial length referenced from the specimen back-end. In both the high and low velocity experiments, the dynamic yield stress is highest at the early stages of deformation (Phase I), but only the specimen tested at $205 \mathrm{~m} / \mathrm{s}$ displays a regime of perfectly plastic response. 


\section{Tables}

Table 1 Time after impact for selected frames, in $\mu$ s

\begin{tabular}{cccccccc}
\hline $\begin{array}{c}\text { Impact } \\
\text { Velocity }\end{array}$ & Frame 1 & Frame 2 & Frame 3 & Frame 4 & Frame 5 & Frame 6 & Frame 7 \\
\hline $83 \mathrm{~m} / \mathrm{s}$ & 20.2 & 32.9 & 45.6 & 58.4 & 71.1 & - & - \\
$205 \mathrm{~m} / \mathrm{s}$ & 5 & 15 & 25 & 35 & 45 & 60 & 90 \\
\hline
\end{tabular}


Figure 1
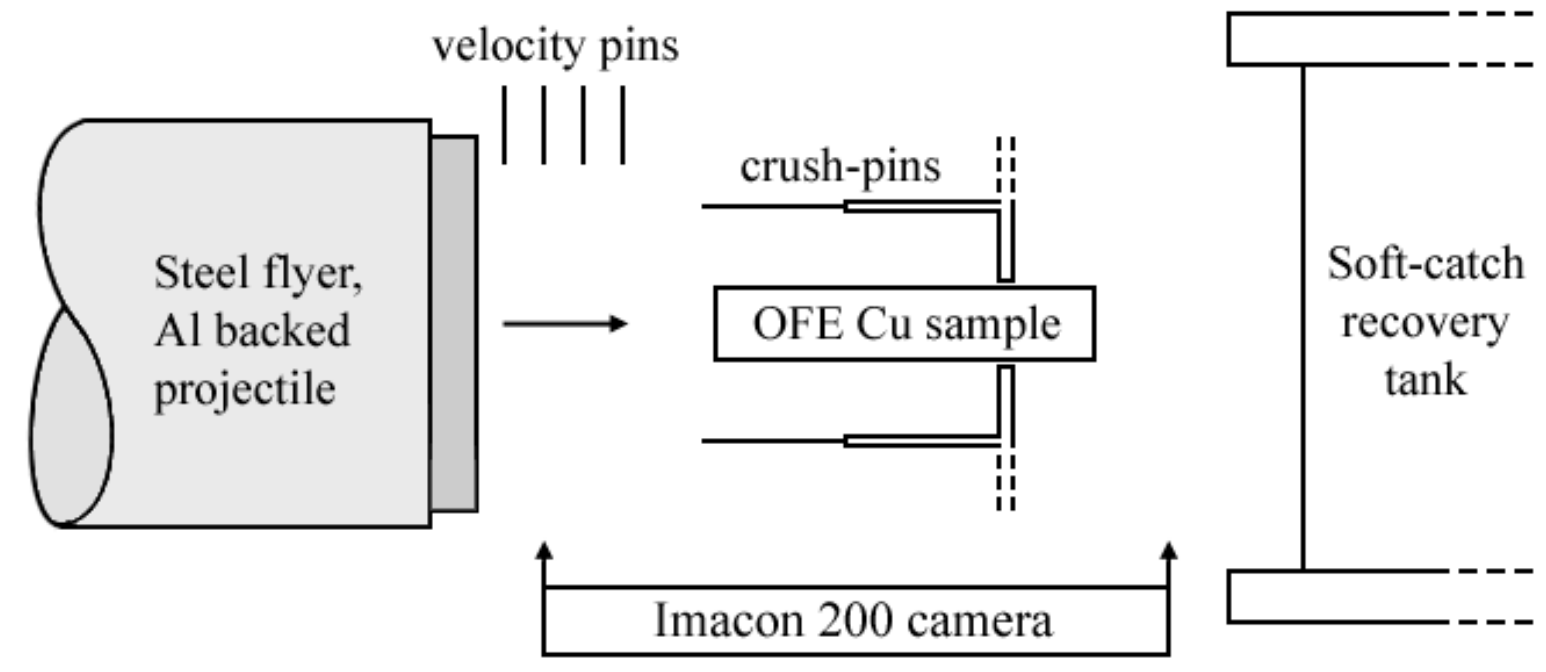
Figure 2

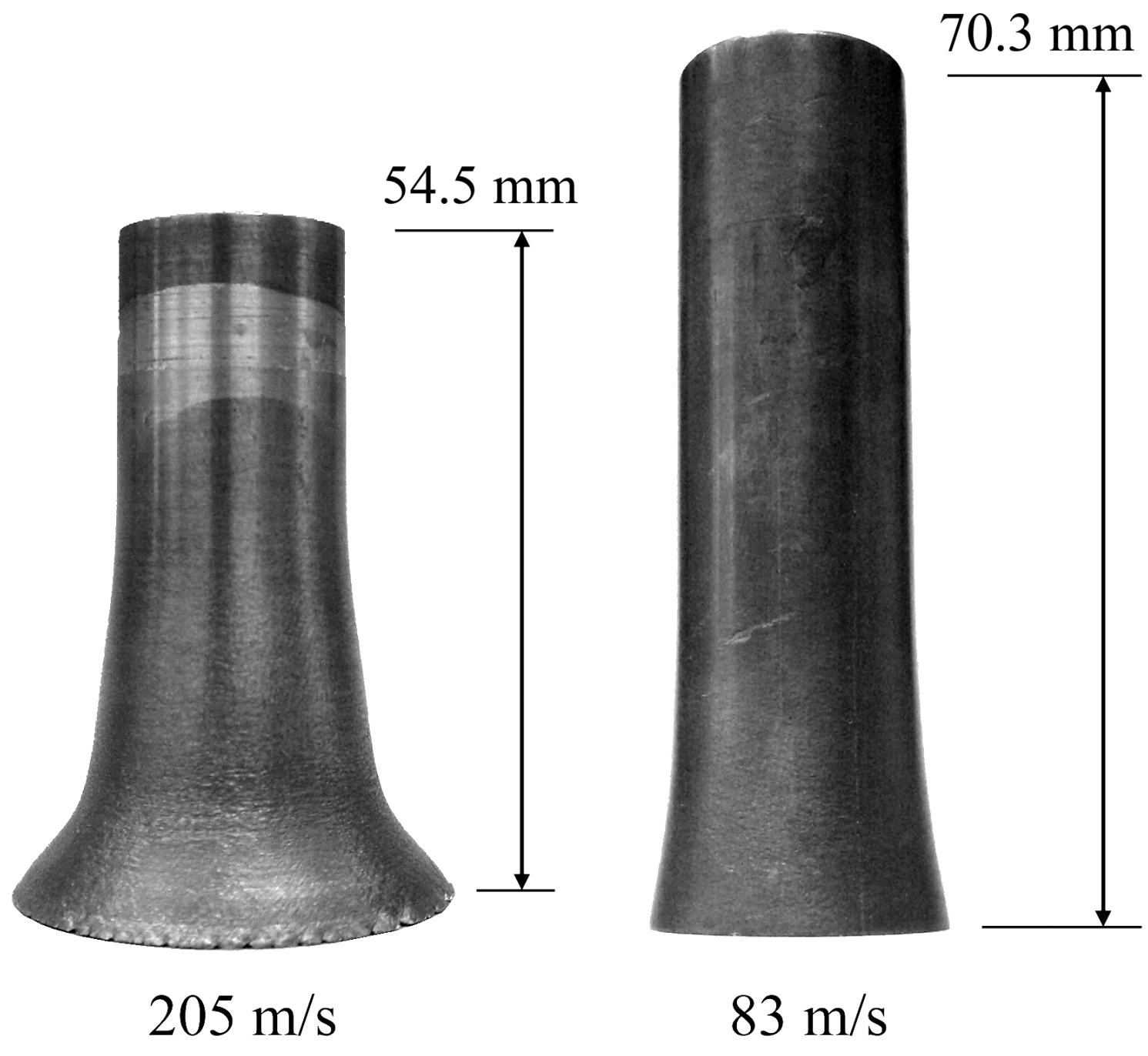


Figure 3
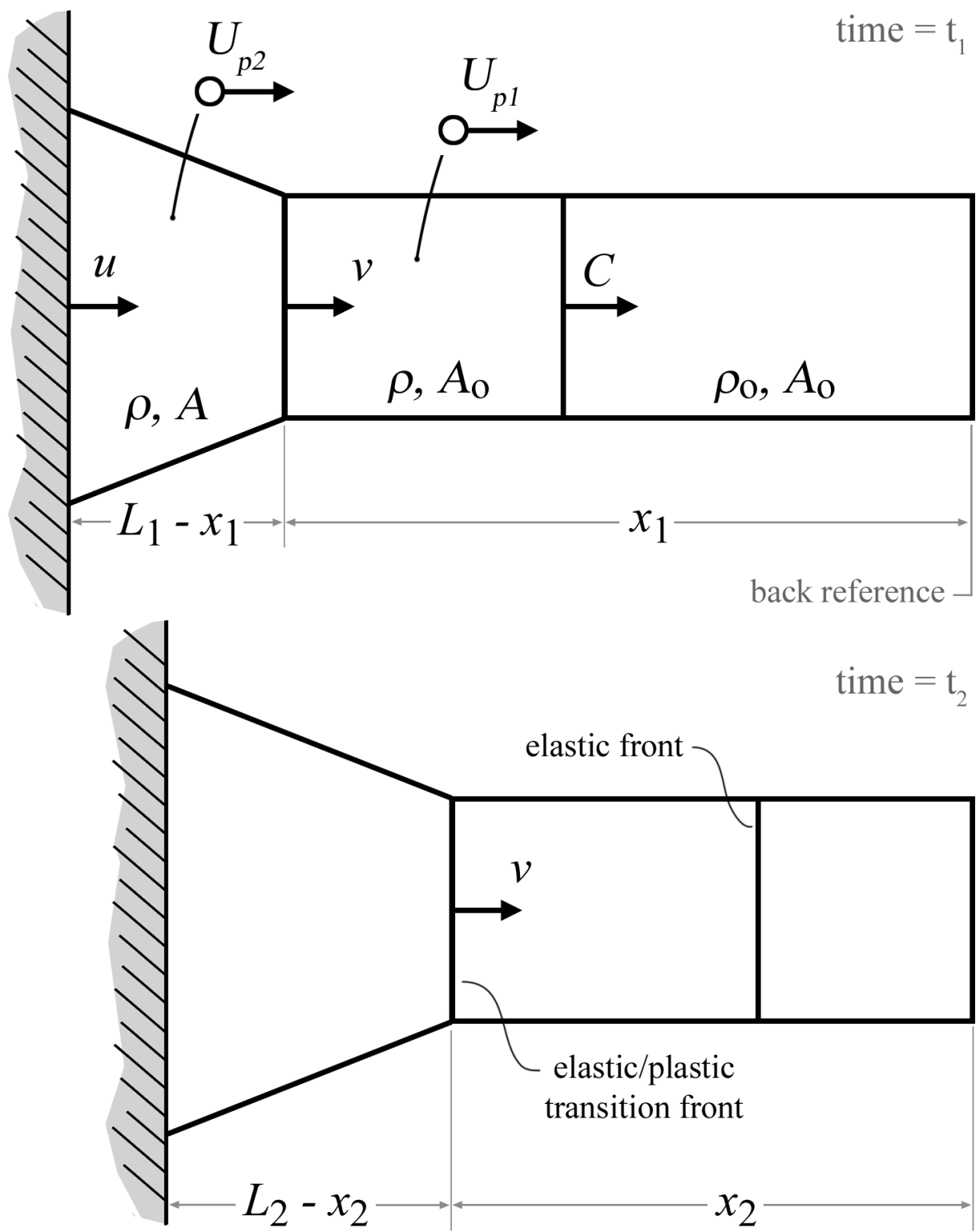
Figure 4

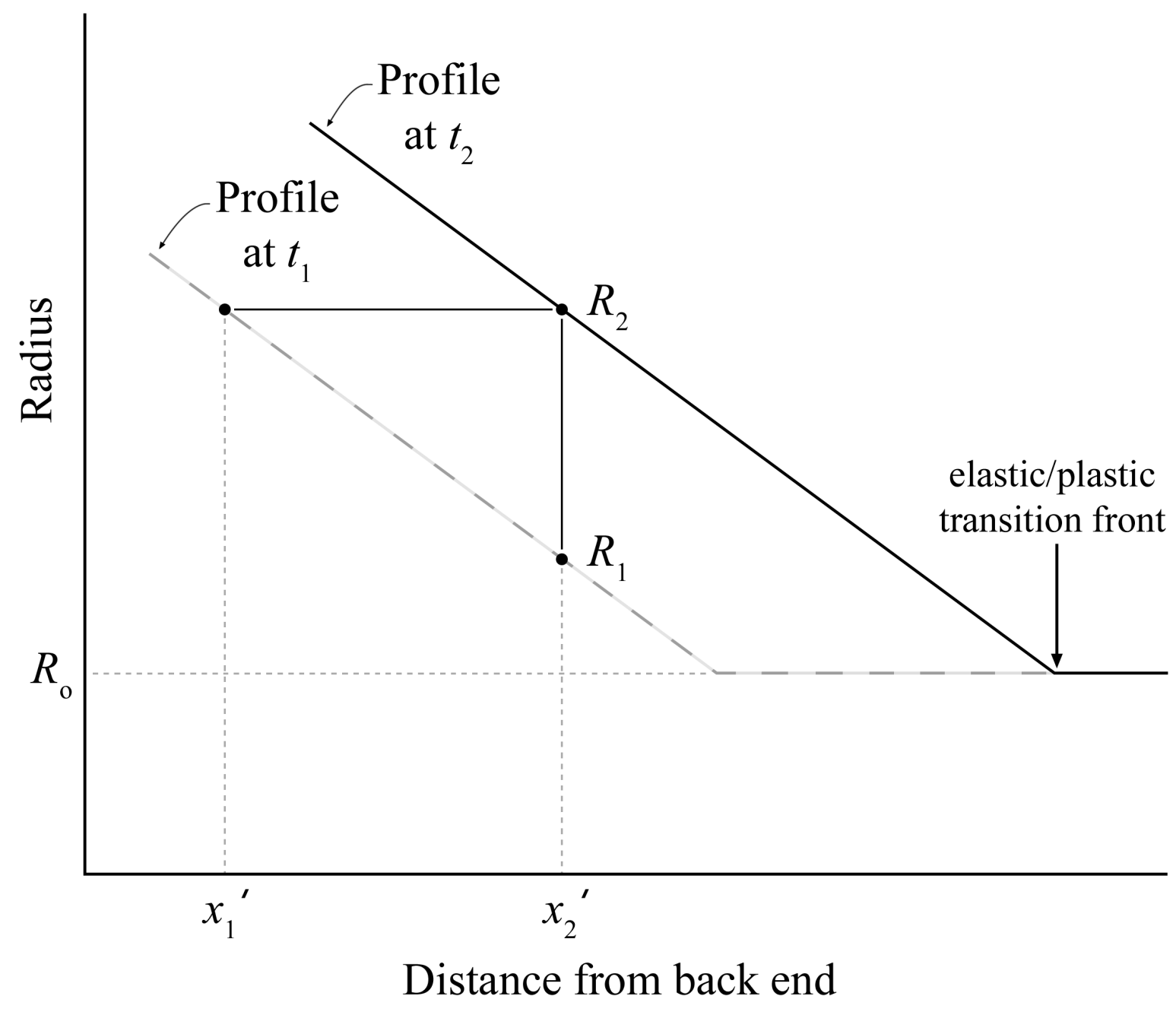


Figure 5

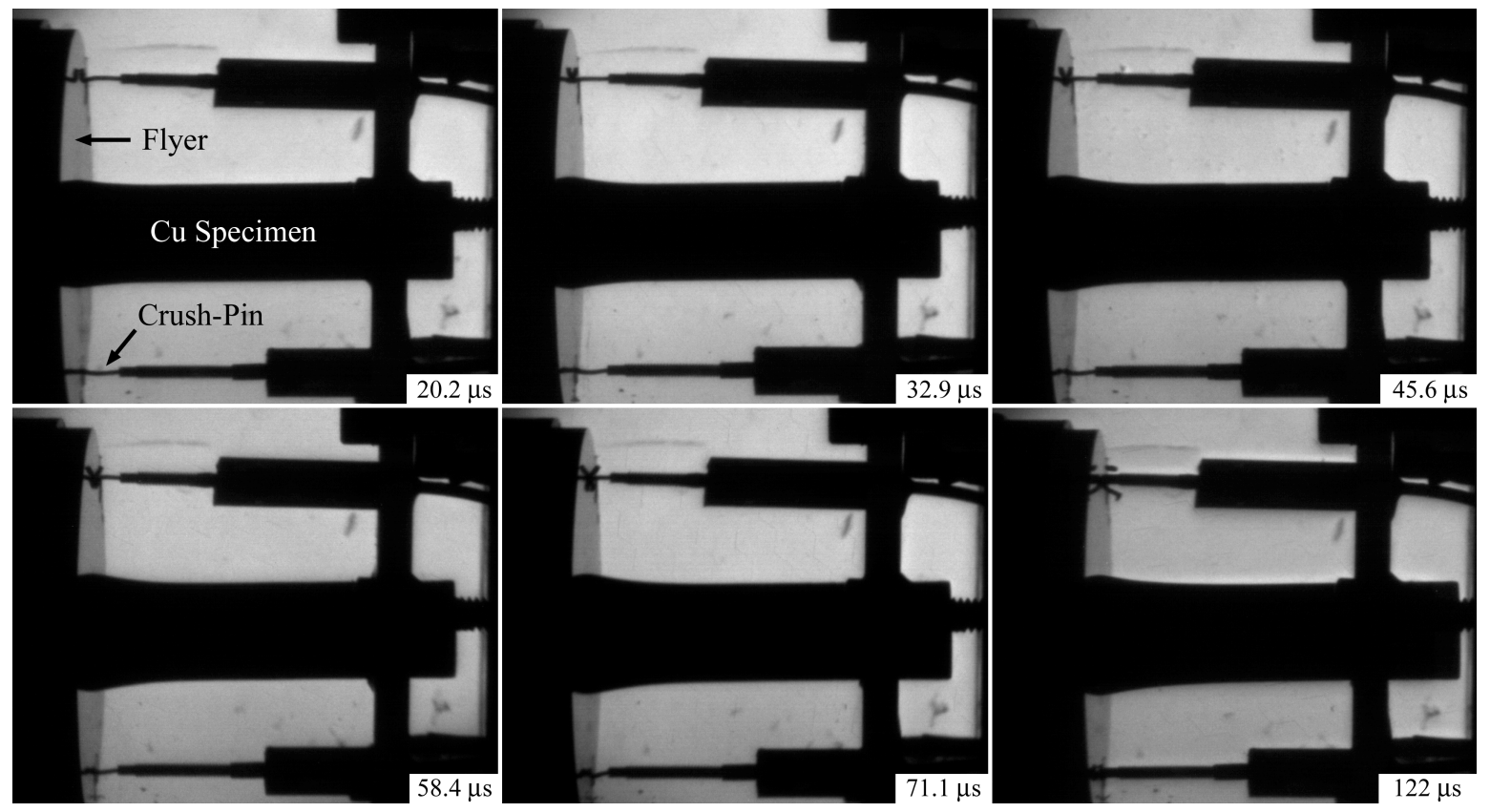


Figure 6

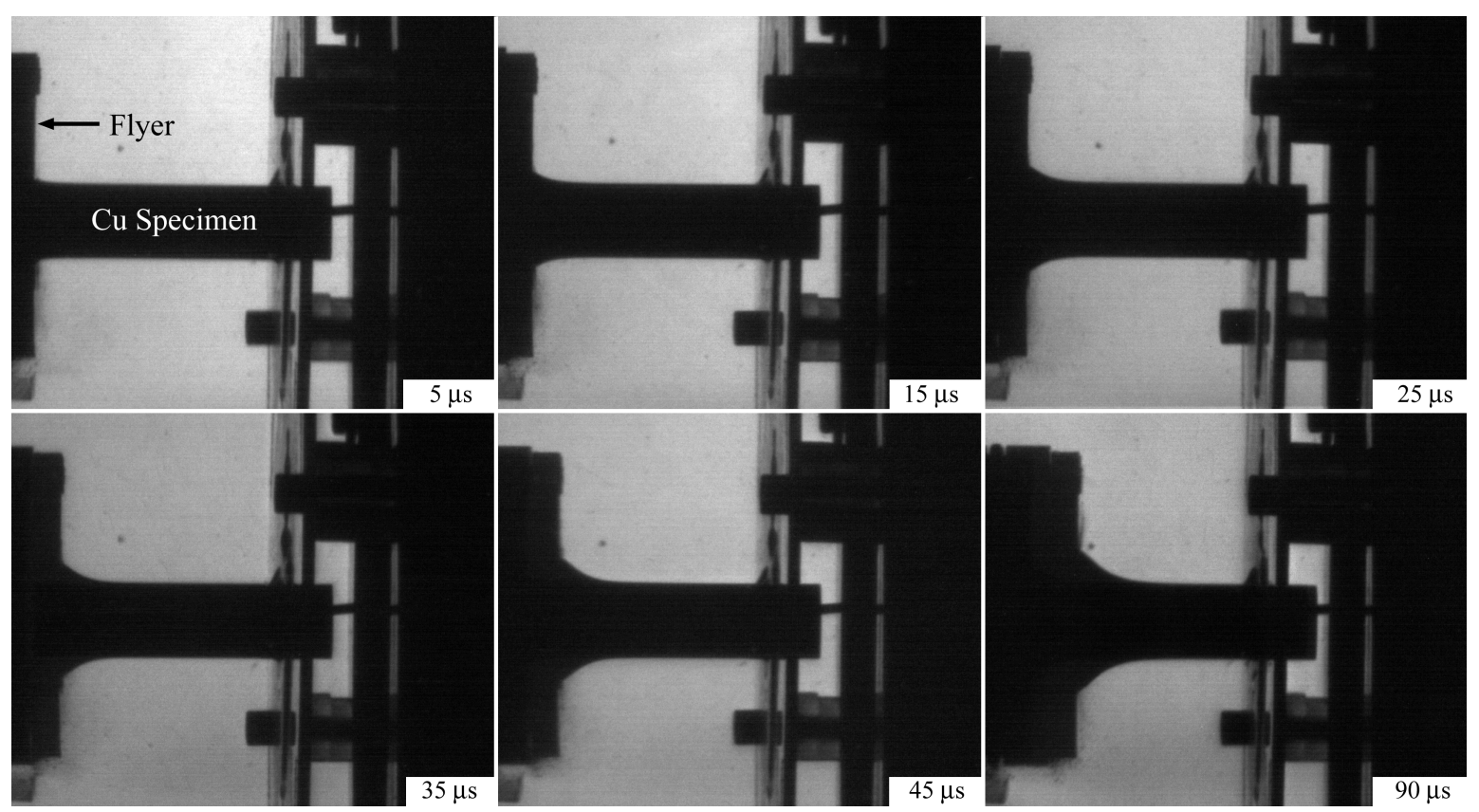


Figure 7
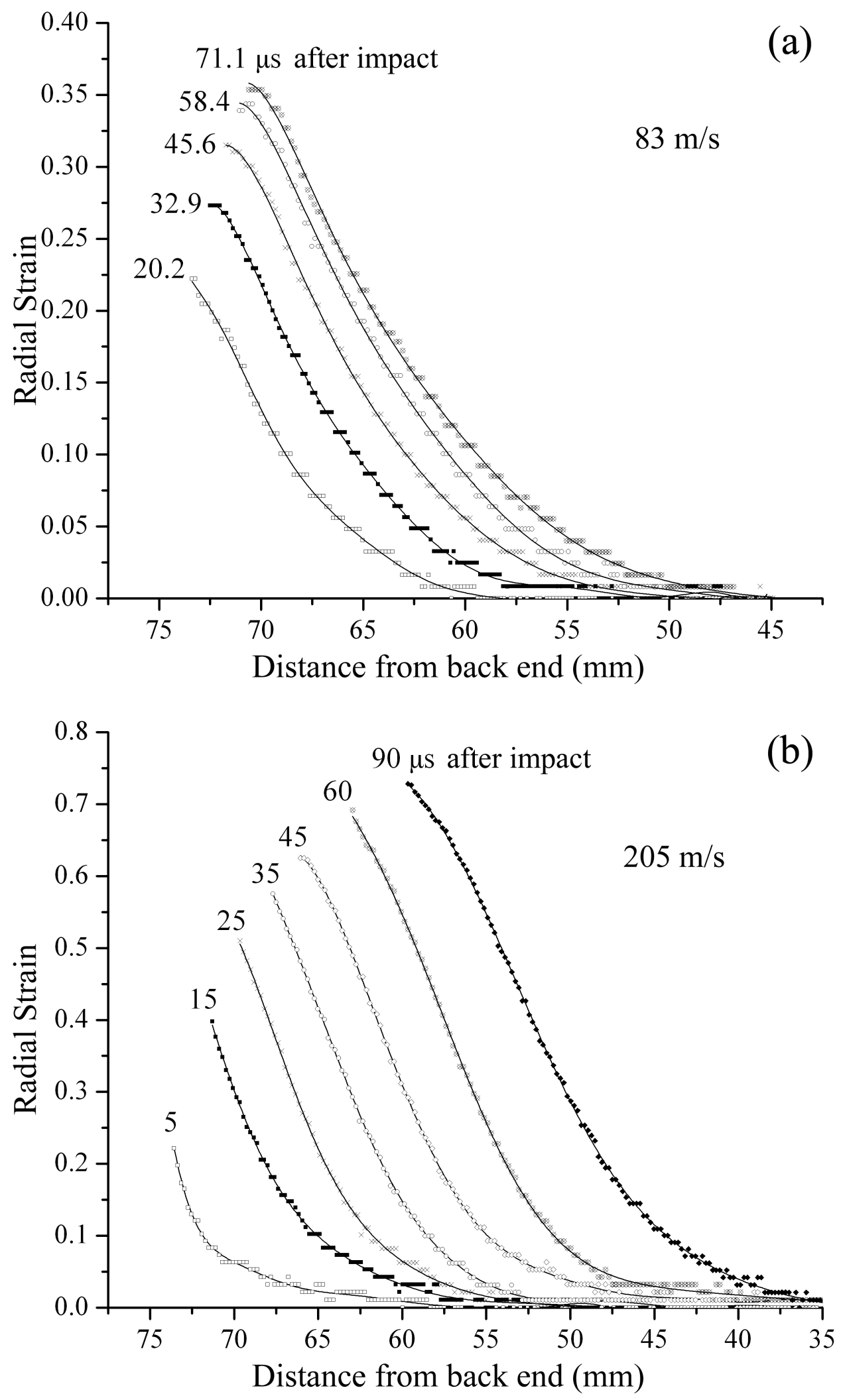
Figure 8

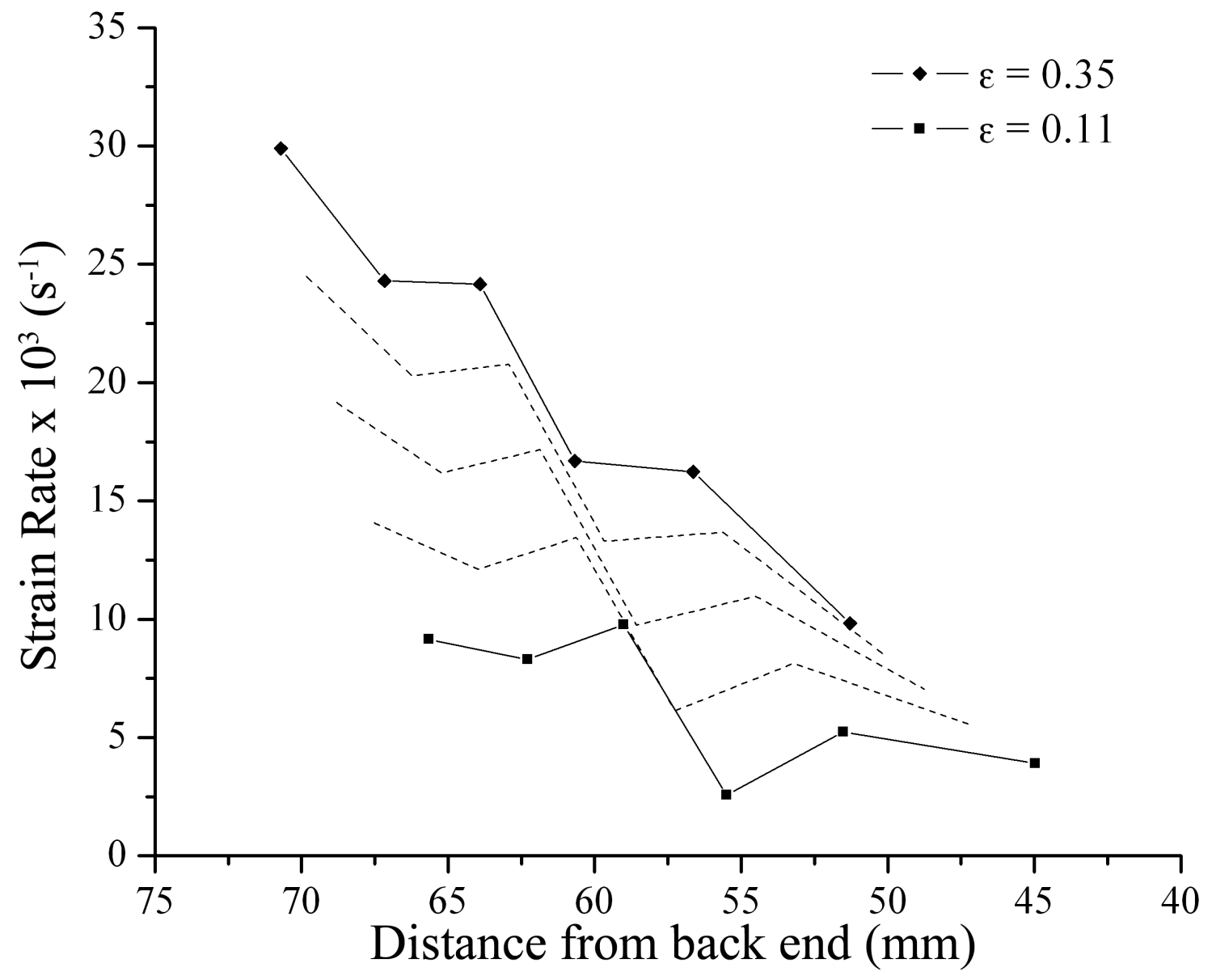


Figure 9

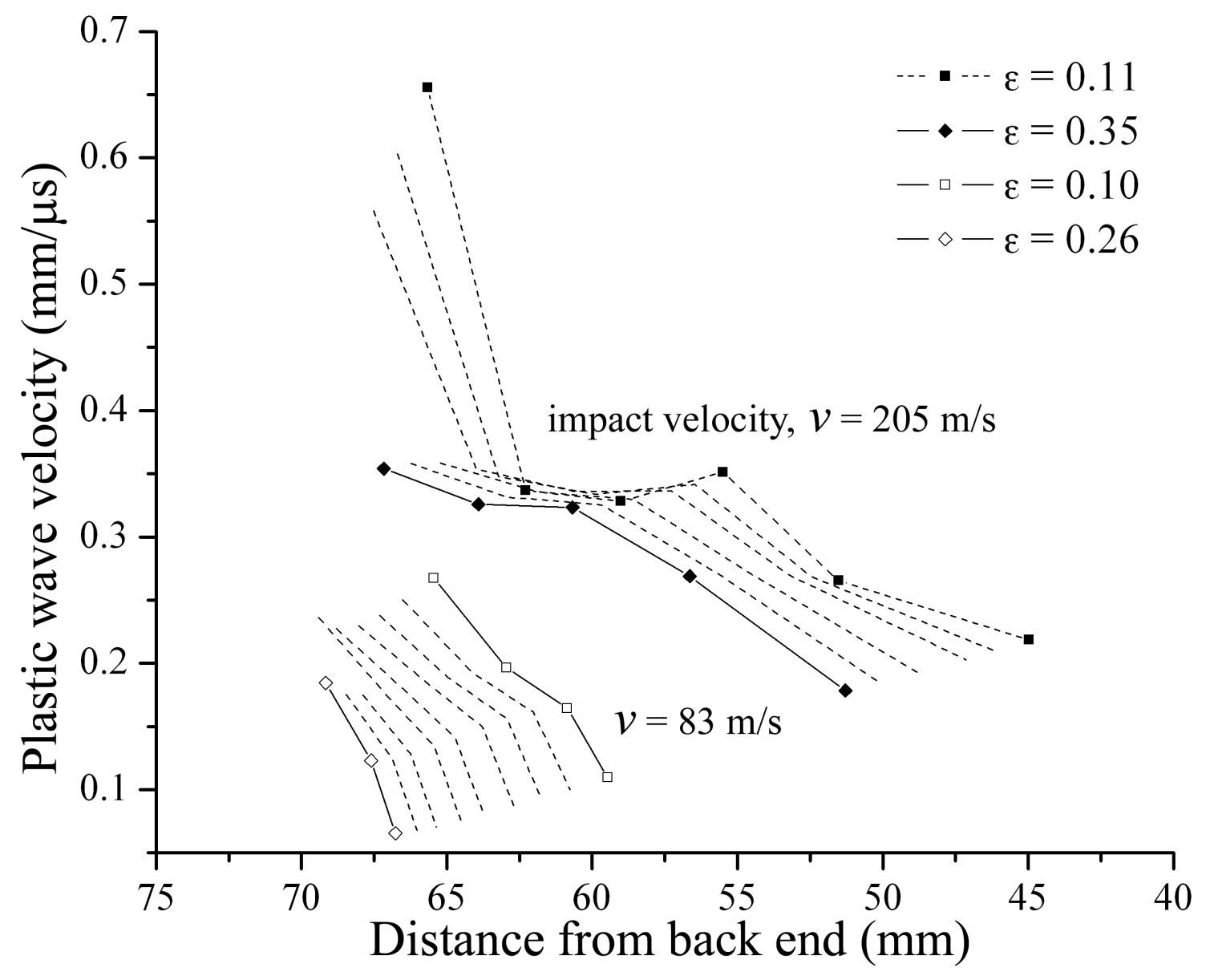


Figure 10

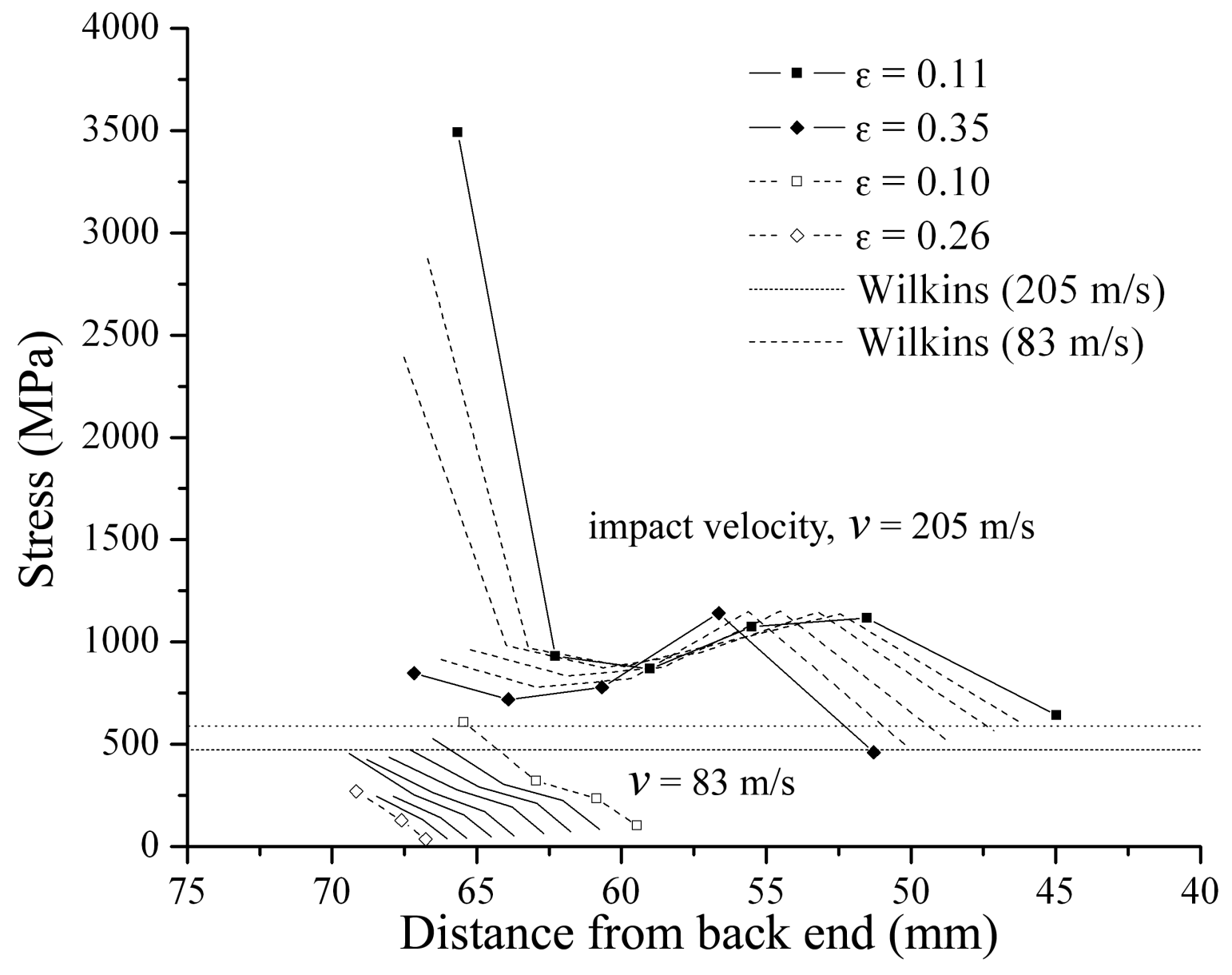

